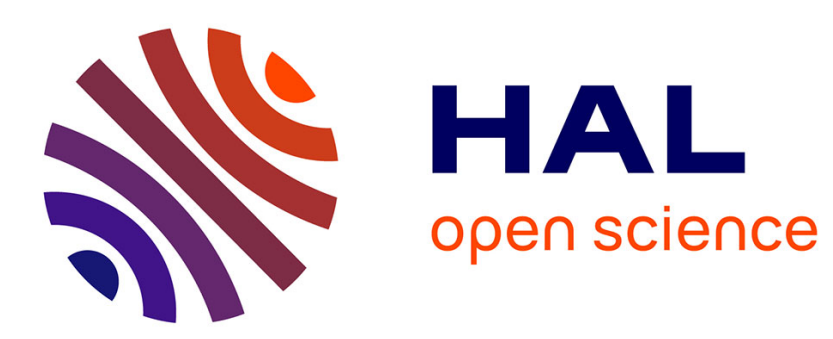

\title{
Évitement à l'entrée en sixième et division sociale de l'espace scolaire à Paris
}

Jean-Christophe François

\section{To cite this version:}

Jean-Christophe François. Évitement à l'entrée en sixième et division sociale de l'espace scolaire à Paris. Espace Géographique, 2002, 4, pp.307 à 327 . halshs-00160710

\section{HAL Id: halshs-00160710 \\ https://shs.hal.science/halshs-00160710}

Submitted on 6 Jul 2007

HAL is a multi-disciplinary open access archive for the deposit and dissemination of scientific research documents, whether they are published or not. The documents may come from teaching and research institutions in France or abroad, or from public or private research centers.
L'archive ouverte pluridisciplinaire HAL, est destinée au dépôt et à la diffusion de documents scientifiques de niveau recherche, publiés ou non, émanant des établissements d'enseignement et de recherche français ou étrangers, des laboratoires publics ou privés. 


\title{
EVITEMENT A L'ENTREE EN SIXIEME ET DIVISION SOCIALE DE L'ESPACE SCOLAIRE A PARIS
}

\begin{abstract}
Résumé :
En France, les élèves sont normalement dirigés vers un établissement scolaire public en fonction de leur adresse de résidence. Une partie des familles évite cet établissement par divers moyens. L'objet de ce travail est de mesurer les effets de cet évitement scolaire sur la division sociale de l'espace scolaire de Paris intra-muros. On constate d'abord que 1 'évitement est pratiqué très inégalement par les différentes catégories sociales, les plus favorisés évitant davantage. Néanmoins, la réussite des demandes de dérogation officielle n'est pas liée, à Paris, à l'appartenance sociale du demandeur. On montre ensuite que l'évitement scolaire renforce de façon significative la division sociale de l'espace parisien, et ce, à différents échelons géographiques. En revanche, les conséquences de l'évitement sur la mixité sociale mesurée en termes de probabilité de contact entre élèves de catégories sociales différentes, demeurent pour l'instant assez limitées. On recherche enfin, dans le contexte social d'une part et le contexte géographique d'autre part, des composantes explicatives de l'évitement.
\end{abstract}

Mots-clefs : ségrégation, enseignement secondaire, Paris, méthodologie

Aborder la question de l'évitement scolaire, c'est, au-delà de ses aspects plus ou moins sensationnels ${ }^{1}$, affronter la problématique de la mixité sociale à l'école, point de mire qui focalise les tensions. Tensions entre utilité individuelle et utilité collective, ou, si l'on préfère, entre volonté d'intégration individuelle et chances d'intégration collective. Tensions entre un enseignement public sectorisé et un enseignement privé qui ne l'est pas. Tensions entre établissements publics en concurrence entre eux malgré la sectorisation pour capter les élèves intéressants (en avance et/ou issus de milieux socialement favorisés).

Dans le contexte français, où les élèves sont normalement scolarisés en fonction de leur lieu de résidence, l'évitement peut être défini comme l'ensemble des pratiques des familles qui scolarisent leurs enfants dans un autre établissement que celui de l'aire de recrutement à laquelle appartient leur domicile. Les motifs de ces familles peuvent être positifs. Il arrive en effet que le collège de secteur laisse la famille indifférente, ou même qu'elle lui prête plus de qualités que de défauts, mais qu'elle estime, à tort ou à raison, que la réussite sociale ou professionnelle de l'enfant passe par sa scolarisation dans un autre établissement jugé plus prestigieux. C'est là un motif très courant d'évitement dans les espaces de réussite scolaire, en particulier à Paris intra-muros. Dans de nombreux cas cependant, le motif de l'évitement est, au moins en partie, négatif. Il s'agit dans ce cas pour la famille d'échapper à l'établissement de secteur, perçu à tort ou à raison comme favorisant ou conditionnant l'échec scolaire et/ou une proximité sociale jugée "dégradante" ou "dangereuse" (on y redoute les "mauvaises fréquentations"). Motivations positives et motivations négatives ne sont ni exclusives, ni toujours clairement formulées.

Quel que soit son motif, l'évitement revêt différentes formes, légales ou illégales, lesquelles sont plus ou moins transparentes pour le chercheur. Du point de vue de l'administration scolaire, l'élève n'a que deux solutions. Ou bien il respecte, bon gré mal gré, la carte scolaire, ou bien il demande (et dans la majorité des cas obtient) une dérogation officielle. Toutes les autres pratiques sont bien connues des acteurs, mais sont de fait ignorées de l'institution qui les regroupe sous le vocable "évaporation". La métaphore semble pertinente: une partie des élèves, en effet, disparaît, "s'évapore" sans laisser de traces. Cette "évaporation", dont l'ampleur est difficile à évaluer, recouvre plusieurs pratiques.

\footnotetext{
${ }^{1}$ Qui dit évitement scolaire dit stratégies, trucs et astuces, secrets de polichinelle jalousement gardés et fausses rumeurs, manne providentielle pour la presse en mal de tirage, marque de distinction sociale, effervescence autrefois réservée aux classes moyennes et supérieures qui agite aujourd'hui toute la société...
} 
Une partie des familles court-circuite l'administration scolaire en traitant directement avec les chefs d'établissement concernés. Cette pratique est assez fréquente dans l'enseignement primaire, mais elle devient exceptionnelle à partir du collège.

Une autre partie des familles triche. Dans le cas d'une famille "pionnière" récemment arrivée dans un quartier dont l'embourgeoisement débute, on observe fréquemment la nondéclaration du changement de résidence: la famille préfère alors, si la distance n'est pas excessive, que les enfants demeurent scolarisés dans son quartier d'origine. Dans le cas de fraude le plus général, la famille "évitante" déclare une fausse adresse justifiant l'inscription des enfants dans un autre collège. On voit ainsi prétendre que les enfants résident chez des parents, des amis qui habitent non loin d'un établissement prestigieux. D'aucuns n'hésitent pas à louer une chambre de bonne, voire un box où ils font installer le téléphone (!) dans le seul but de produire la quittance attestant que l'élève réside bien dans le secteur de l'établissement convoité. Le désir d'évitement peut même être un critère décisif dans la décision de déménager. Il est clair que ces manœuvres sont réservées à des familles disposant de moyens financiers non négligeables. Enfin, le désir d'évitement motive bon nombre des transferts de l'enseignement public à l'enseignement privé, qui n'est pas sectorisé.

Parfois présente dès la crèche, l'angoisse des parents devant le choix de la «bonne école ${ }^{2}$ » croît avec l'enfant. L'entrée en sixième constitue à cet égard un palier d'une importance toute particulière: la mixité sociale au sein de l'établissement scolaire semble beaucoup moins bien tolérée par les parents les plus favorisés dès lors que leur enfant entre au collège (PINCON et PINCON-CHARLOT, 1992).

\section{Les données}

La population étudiée est celle des élèves qui sont entrés en $6^{\mathrm{e}}$ à la rentrée 2001 , à l'échelon géographique de l'établissement - ou, plus exactement, de l'aire de recrutement de celui-ci. Les données mobilisées ici distinguent quatre catégories d'élèves selon leur appartenance sociale: «Favorisé A», «Favorisé B», «Moyen», «Défavorisé». Ces catégories sont construites par l'administration scolaire à partir de la profession déclarée par le père ou le responsable légal de l'enfant. Si elles ne constituent pas une grille aussi précise que celles du RGP, on peut cependant leur reconnaître une pertinence suffisante ici pour deux raisons. Premièrement, les risques d'erreurs ou d'artefacts statistiques liés à la division de la population en souspopulations ne sont pas négligeables quand on travaille sur des effectifs de l'ordre d'une centaine d'élèves de sixième par établissement (effectif moyen des aires de recrutement: 130). Ils sont limités si on accepte de se contenter des quatre catégories de l'Education nationale au lieu des huit proposées au minimum par l'INSEE. Deuxièmement, ces catégories propres à l'administration scolaire sont construites en tenant compte non seulement de la position économique, mais aussi du «capital scolaire»supposé de chaque profession. Ainsi par exemple les enfants d'instituteurs sont «surclassés» dans la catégorie «Favorisés A», alors que les enfants des professions intermédiaires administratives de la fonction publique sont, eux, rangés dans la catégorie «favorisés B ». En ce qui concerne l'évitement scolaire, cette classification prenant en compte le «capital scolaire» (directement, par l'accès à l'information sur le système scolaire, indirectement, via les préjugés sur la capacité à réussir des enfants des différents milieux en fonction de leur classement dans la grille de l'Education Nationale...) peut s'avérer pertinente. C'est pourquoi, tout en s'appuyant sur des grilles plus fines pour d'autres recherches concernant l'espace scolaire, il a semblé possible de travailler ici à partir de ces quatre catégories. Pour chacune de ces catégories, on connaît le nombre d'élèves du secteur scolarisés en CM2, et, parmi eux, le nombre de ceux qui choisissent le

\footnotetext{
${ }^{2}$ Langouet \& Morvan, 1991
} 
privé. On connaît également l'effectif de chaque catégorie en classe de sixième après le mouvement des dérogations ${ }^{3}$.

Muni de ces données, on se pose les questions suivantes :

-Les différentes catégories sociales sont-elles inégales devant l'évitement?

-Quel est l'effet de l'évitement sur l'espace scolaire ? Quelles sont ses conséquences sur les effectifs des établissements, sur leur structure sociale, sur le niveau de ségrégation ?

-Comment expliquer l'inégale répartition spatiale de l'évitement? Quels sont les rôles respectifs de la structure sociale de l'établissement évité, de l'évolution de celle-ci, de la structure sociale des attracteurs potentiels que sont les établissements voisins ?

\section{L'évitement scolaire en 2001}

\subsection{Spécificités parisiennes}

L'espace scolaire parisien se distingue d'abord par la très grande diversité de l'offre scolaire. La forte densité de la population induit une forte densité du semis des établissements publics et privés, la centralité renforce l'offre en induisant la localisation intra-muros d'établissement atypiques ou spécialisés, elle induit également une bonne accessibilité des établissements scolaires par les transports en commun.

Il se distingue ensuite par son profil social atypique, qu'on peut résumer en trois points: une forte présence des catégories favorisées, une différenciation sociale de l'espace forte et ancienne entre un ouest bourgeois et un nord-est populaire, avec un mouvement important d'embourgeoisement de ce dernier.

Il se distingue enfin par l'importance et l'ancienneté des pratiques d'évitement. Si l'on compare la situation de 2001 avec celle de 1992, on constate que la propension à demander une dérogation à l'entrée en sixième n'a que très légèrement augmenté ces dernières années ( $16 \%$ des élèves de $6^{\mathrm{e}}$ en 2001 contre $15 \%$ en 1992). La politique «d'assouplissement » du début des années 1990 ayant fait place à un retour à une sectorisation stricte (mono établissement), le taux de satisfaction global des demandes est aujourd'hui nettement plus bas ( $49 \%$ contre $81 \%)$.

La procédure de dérogation, établie par le Ministère, est en principe commune à tous les départements français. Cependant, la relative autonomie dont jouissent les Inspecteurs d'Académie en la matière entraîne des nuances importantes, non seulement dans la proportion des décisions favorables, mais encore dans l'organisation de la procédure elle-même. La présentation même du dossier de demande de dérogation rempli par la famille peut varier sensiblement d'un département à l'autre. A Paris, onze motifs sont proposés sur le formulaire de dérogation: Santé, Langue vivante, fratrie dans le collège, parents travaillant dans l'établissement, proximité du domicile, horaires aménagés, établissement "international ", fratrie dans le lycée, proximité du travail des parents, hébergement dans le secteur de l'établissement désiré, autres.

\footnotetext{
${ }^{3}$ On voit que l'information disponible ici se limite à la partie officielle de l'évitement (dérogations et départs vers le secteur privé). On ne mesure donc ici qu'une partie du décalage entre l'espace scolaire et l'espace de résidence.
} 


\subsection{L’évitement, un phénomène socialement différencié}

Les taux d'évitement (fig.1) suivent exactement la hiérarchie sociale, les plus favorisés évitant davantage. Si les taux d'évitement des « défavorisés » sont toujours très inférieurs à ceux des autres catégories, cette différence est plus marquée pour le recours au privé que pour les dérogations. En revanche, parmi les élèves «favorisés », c'est le recours à la dérogation qui est le plus discriminant: c'est par les dérogations que les élèves «favorisés A » se distinguent le plus. Le filtrage social s'effectue donc d'abord en amont, par une inégale propension des différentes catégories à éviter.

Fig.1 Le taux d'évitement est fonction de la catégorie sociale

\begin{tabular}{|l|l|l|l|l|l|}
\hline $\begin{array}{l}\text { propension à choisir } \\
\text { le privé au moment } \\
\text { de l'entrée en 6 }\end{array}$ & 0.09 & «Favorisés B » & « Moyens » & "Défavorisés » & Toutes catégories \\
\hline $\begin{array}{l}\text { propension à } \\
\text { demander ù } \\
\text { dérogation à la carte } \\
\text { scolaire } \\
\text { au moment de } \\
\text { l'entrée en 6 }\end{array}$ & 0.22 & 0.08 & 0.07 & 0.02 & 0.06 \\
\hline $\begin{array}{l}\text { Propension à éviter* } \\
\text { au moment de } \\
\text { l'entrée en 6 }\end{array}$ & 0.31 & 0.19 & 0.16 & 0.10 & 0.16 \\
\hline
\end{tabular}

* formes illicites non comprises

\section{3. la réussite des différentes catégories pour l'obtention d'une dérogation}

Le taux de satisfaction global, toutes catégories confondues, est assez élevé $(49 \%$ des demandes), mais il est très inégal: selon le motif invoqué, les chances de réussite de la demande varient considérablement (de 19 à plus de 89 sur cent).

Que penser des motifs déclarés ? Ceux-ci ne sont-ils que des prétextes ? Dans la mesure où l'on n'observe aucune demande de sortie des établissements les plus prestigieux, on peut estimer que les demandes trahissent toujours, quel que soit le motif invoqué, des velléités d'évitement ${ }^{4}$. On peut donc faire l'hypothèse qu'il existe des stratégies différenciées selon l'appartenance sociale, les catégories les plus favorisées connaissant les motifs les plus efficaces pour obtenir une dérogation. Pour le vérifier, on dispose ici d'une catégorisation sociale plus précise des élèves parisiens (28 catégories socioprofessionnelles décrivant, tous collèges publics confondus, la population des élèves demandant et obtenant une dérogation). L'amplitude du taux de satisfaction des demandes de dérogation selon la catégorie socioprofessionnelle des parents est remarquablement faible. Celui ci va de $47 \%$ (artisans, commerçants et entrepreneurs) à 53\% (techniciens, agents de maitrise) Contre toute attente, les demandes des cadres supérieurs aboutissent moins que celles des ouvriers, avec respectivement $49 \%$ et $51 \%$ de demandes satisfaites. Plus surprenant encore, un examen plus détaillé fait apparaître des taux de satisfaction très médiocres pour les enfants de professeurs (48\%) et à peine supérieurs à la moyenne pour les enfants d'instituteurs.

\footnotetext{
${ }^{4}$ On trouve régulièrement dans la presse des conseils pour « réussir sa dérogation » en indiquant «le bon motif ». Cf en annexe un article édifiant d'un magazine destiné « aux familles ».
} 


\section{Il semble donc qu'à Paris, l'appartenance sociale du demandeur n'influence pas significativement les chances de succès de la dérogation.}

La raison principale de ce résultat contre-intuitif doit être recherchée dans la procédure mise en place par l'Académie: pour chaque établissement, les demandes invoquant le même motif sont considérées en bloc, en fonction des places disponibles. L'administration scolaire va décider par exemple, dans un établissement donné, de satisfaire ou de rejeter toutes les demandes d'inscription motivées par le choix d'une langue vivante. Les pressions individuelles en sont minimisées, et les «stratégies » individuelles d'évitement par la dérogation plus difficile à mettre en place.

Toutefois, le nombre de places disponibles étant pris en compte par l'administration, il résulte qu'il est globalement plus difficile d'accéder à un établissement très demandé. Par ailleurs, les aires de recrutement de certains établissements comprennent une population résidante trop peu nombreuse, permettant «l'immigration » d'un certain nombre d'élèves résidant dans d'autres secteurs. Il conviendrait donc de confirmer cette indépendance entre la réussite de la dérogation et l'appartenance sociale du demandeur en disposant des mêmes données à l'échelon des établissements; il est possible en effet que l'homogénéité des taux de satisfaction dissimule des demandes de nature différentes : les catégories supérieures seraient plus ambitieuses, demandant des établissements particulièrement difficiles à obtenir (où les places sont rares), tandis que les autres auraient tendance à limiter leurs ambitions. Il faudrait également prendre en compte l'accessibilité géographique des établissements les plus côtés, dont les élèves défavorisés sont $l$ loin $^{5}$. Enfin, il convient de ne pas minimiser l'évitement illégal et l'évitement précoce ${ }^{6}$, qui ne sont pas pris en compte ici.

\section{Les effets de l'évitement sur l'espace scolaire}

\subsection{Evitement et effectifs des établissements}

Dans la mesure où la propension à déroger est liée à l'appartenance sociale de l'élève, on pourrait s'attendre à ce que la carte des dérogations reproduise peu ou prou la carte bien connue des disparités sociales à Paris intra-muros ; or, il n'en est rien. Néanmoins, malgré l'apparente complexité de la carte obtenue (fig.2a), on peut repérer quelques traits majeurs:

Tout d'abord, l'espace scolaire parisien se structure autour d'un pôle attractif principal situé dans le centre-ouest. Cette localisation coïncide certes avec les beaux quartiers, mais ne les englobe pas complètement, loin de là. Les établissements les plus prestigieux du centre parisien, ceux de la rive gauche notamment, s'il sont généralement attractifs, le sont beaucoup moins qu'on pouvait s'y attendre. Le pôle attractif principal est nettement décentré vers l'ouest. Il est également peu cohérent spatialement (un établissement du VIIe arrondissement perd même des élèves).

\footnotetext{
${ }^{5}$ Ce qu'on sait de la portée géographique des demandes semble conforter ces hypothèses.

${ }^{6}$ Les dérogations à l'entrée en sixième ne prennent pas en compte les dérogations précoces, celles qui ont lieu dès l'école primaire (ou même parfois dès l'école maternelle). Or, celles-ci concernent une partie non négligeable des élèves. Ainsi telle aire de recrutement où d'après l'Inspecteur de l'Education Nationale "deux élèves sur cinq ont déjà dérogé en primaire".Toutefois, l'importance des pratiques d'évitement souterraines ou illicites ne remet pas en cause la validité de l'indicateur pour la suite de ce travail. On peut en effet sans trop de risque d'erreur supposer que l'évitement global est proportionnel à la partie officiellement enregistrée. En d'autres termes, on peut penser que là où il existe un flux important de dérogations, l'évitement dans son ensemble est massif.
} 
Autour de ce pôle principal, un chapelet de petits pôles régulièrement espacés sont constitués chacun d'un établissement isolé. Leur absence rive gauche peut s'expliquer par la concurrence des établissements prestigieux du centre, relativement proches et accessibles.

Cette régularité dans la localisation des pôles attractifs pourrait s'expliquer par les échelons géographiques de la concurrence entre établissements: hormis les établissements prestigieux du centre, dont l'offre scolaire « haut de gamme » dilate l'aire d'attraction, le recrutement des établissements est principalement local. On trouve donc une structuration de l'espace scolaire en lieux centraux hiérarchisés qui ressemble dans une certaine mesure à celle qui est observable dans le cas des services marchands. Elle en diffère cependant en ce que le pôle principal repéré ci-dessus n'est pas le lieu le plus attractif. Cette place de choix revient en effet à deux établissements formant des pôles attractifs isolés et situés de part et d'autre de celui-ci (Porte d'Auteuil pour l'un, quartier Saint-Georges pour l'autre. Moins prestigieux que certains de leurs voisins immédiats, ils compensent ce handicap par l'extension importante de leurs aires d'attraction: celles-ci englobent plusieurs arrondissements et la proche banlieue desservie par le métro (Boulogne-Billancourt pour l'un, Clichy et Saint-Ouen pour l'autre).

Autour de ces pôles plus ou moins attractifs, on trouve toute une palette d'établissements aux comportements très divers, depuis les établissements dont les effectifs sont peu modifiés jusqu'aux aires de recrutement qui perdent le plus d'élèves entre le CM2 et la $6^{\mathrm{e}}$. Les établissements les plus répulsifs sont fréquemment situés en périphérie, mais l'espace scolaire situé au-delà des boulevards extérieurs est loin d'être homogène en matière de flux de dérogations, même au nord est.

La carte de l'effet des départs vers le privé (Fig.2b) présente une configuration spatiale particulière : certains établissements centraux voient beaucoup d'élèves partir vers le privé au moment de l'entrée en sixième (premier arrondissement), d'autres pas du tout (c'est le cas des établissements du $5^{\mathrm{e}}$ ). La périphérie présente des contrastes importants, avec trois pôles répulsifs principaux comprenant chacun une palette de situations très différentes. Le plus compact de ces «pôles répulsifs » se situe au nord de la capitale ( $18^{\mathrm{e}}$ arrondissement). A l'est et au sud-ouest, on a plutôt des grappes d'établissements plus ou moins répulsifs. On observe des comportements contre-intuitifs, notamment sur le pourtour du pôle attractif repéré fig.2a, où des établissements qui connaissent un flux d'évitement important vers le privé sont simultanément attractifs en matière de dérogation. Le XVe arrondissement constitue un exemple particulièrement frappant, avec notamment un établissement qui non seulement se révèle très attractif par voie de dérogation, mais encore accueille des élèves en situation de réussite scolaire (il compte par exemple parmi les établissements où l'avance scolaire est la plus répandue). Pourtant, cet établissement voit près de $20 \%$ des résidents de son aire de recrutement choisir le privé. On ne peut retenir ici l'hypothèse d'un choix du secteur privé pour des motifs étrangers à l'évitement scolaire (choix d'une éducation religieuse par exemple), dans la mesure où, rappelons-le, on ne comptabilise ici que les départs vers le privé au moment de l'entrée en sixième. L'hypothèse d'un lien de cause à effet entre l'attractivité par dérogations et la fuite vers l'enseignement privé est ici plus vraisemblable. La dérogation permet en effet à nombre d'élèves issus de quartiers moins huppés (notamment du sud de l'arrondissement) d'accéder à l'établissement. Cette mixité sociale (modeste au demeurant) n'est pas du goût de toutes les familles; pour peu que l'offre locale du secteur privé soit jugée suffisante -et c'est le cas ici - la proximité sociale redoutée est facilement évitée. Ces mouvements «en cascade », symptomatiques d'un refus de la mixité sociale par de nombreux parents, ont déjà repérés auparavant au sein du seul secteur public (François, 1995 ; Tourret, 1997). Ils amoindrissent beaucoup les effets positifs de l'évitement sur les chances d'intégration individuelle. Un tout autre mécanisme est simultanément à l'œuvre, reposant sur l'échec scolaire des élèves évitants. Le secteur privé, en effet, est souvent un recours en cas 
d'échec (Langouet \& Morvan, 1991); dans un environnement socio-spatial favorisé, un établissement public exigeant ne voit pas toujours d'un mauvais œil partir vers le privé les élèves dissuadés par le niveau élevé qu'il affiche.

Quand on observe (Fig.2c) l'effet combiné des dérogations et du recours au privé, on retrouve, accentuée, la configuration déjà vue fig.2a. Les petits pôles attractifs de l'est, affaiblis par les départs vers le privé, ne sont plus distingués. On y constate seulement le maintient de l'effectif, l'immigration par dérogation équilibrant l'émigration vers le privé. En revanche, on voit apparaître beaucoup plus nettement une couronne périphérique généralement répulsive nettement séparée du centre par une discontinuité significative coïncidant avec l'ancienne enceinte des Fermiers Généraux.

Les effets de l'évitement sur l'effectif des établissements s'exercent donc d'une manière très inégale selon les lieux : qu'en est-il d'éventuels effets sur leur structure sociale?

\subsection{Evitement et structure sociale des établissements}

fig. 3 Composition sociale des collèges publics parisiens

\begin{tabular}{|l|l|l|l|}
\hline $\begin{array}{l}\text { Catégorie } \\
\text { d'élèves }\end{array}$ & $\begin{array}{l}\text { Part dans } \\
\text { l'effectif de } \\
6 \text { e ... }\end{array}$ & $\begin{array}{l}\text { (sans évitement } \\
\text { (situation hypothétique) }\end{array}$ & $\begin{array}{l}\text {...Après évitement } \\
\text { (situation réelle) }\end{array}$ \\
\hline Favorisés A & 0.304 & 0.295 \\
\hline Favorisés B & 0.140 & 0.137 \\
\hline Moyens & 0.258 & 0.257 \\
\hline Défavorisés & 0.298 & 0.310 \\
\hline Total & 1.000 & 1.000 \\
\hline
\end{tabular}

Si l'on observe quel est, globalement, l'impact de l'évitement sur la structure sociale des établissements, on voit (fig.3) que la part relative des élèves «favorisés A » diminue, tandis que celle des élèves « défavorisés » augmente au point qu'ils deviennent, après évitement, la catégorie majoritaire. Ces changements de la composition sociale ne sont pas dus aux dérogations (celles-ci ont lieu pour la plupart intra-muros, et le solde avec la banlieue est positif : Paris est importateur, et, qui plus est, importateur d'élèves favorisés) mais sont évidemment liés à la propension à recourir au secteur privé, propension qui varie selon les catégories d'élèves considérées (de $2 \%$ pour les «défavorisés » à plus de $9 \%$ pour les «favorisés A). A Paris, un peu plus de $6 \%$ des élèves du public se dirigent vers le secteur privé au moment de l'entrée en $6^{\mathrm{e}}$. Sur cent de ces élèves, 44 sont "Favorisés A », 18 sont «Favorisés B », 28 « Moyens » et 10 seulement « défavorisés ».

Fig.4 l'inégal évitement des différentes catégories d'élèves

\begin{tabular}{|l|l|l|l|l|l|}
\hline & $\mathrm{A}$ & $\mathrm{B}$ & $\mathrm{M}$ & $\mathrm{D}$ & $\begin{array}{l}\text { Total } \\
\text { categories }\end{array}$ \\
\hline $\begin{array}{l}\text { Nombre d'élèves } \\
\text { entrant en 6 }\end{array}$ & 4195 & 1929 & 3563 & 4103 & 13790 \\
\hline Proportion d'élèves & 0.304 & 0.140 & 0.258 & 0.298 & 1.000 \\
\hline $\begin{array}{l}\text { Nombre de } \\
\text { dérogations } \\
\text { Demandées }\end{array}$ & 932 & 358 & 572 & 418 & 2280 \\
\hline $\begin{array}{l}\text { Part du volume total } \\
\text { des dérogations } \\
\text { demandées pour } \\
\text { chaque catégorie }\end{array}$ & 0.409 & 0.157 & 0.251 & 0.183 & 1.000 \\
\hline Propension à déroger & 0.221 & 0.186 & 0.161 & 0.101 & 0.165 \\
\hline
\end{tabular}




\begin{tabular}{|l|l|l|l|l|l|}
\hline $\begin{array}{l}\text { Nombre de départs } \\
\text { vers le privé }\end{array}$ & 384 & 161 & 247 & 94 & 886 \\
\hline $\begin{array}{l}\text { Part du volume total } \\
\text { des départs pour } \\
\text { chaque catégorie }\end{array}$ & 0.433 & 0.182 & 0.279 & 0.106 & 1.000 \\
\hline $\begin{array}{l}\text { Propension à recourir } \\
\text { au privé }\end{array}$ & 0.091 & 0.083 & 0.069 & 0.023 & 0.064 \\
\hline $\begin{array}{l}\text { Propension à déroger } \\
\text { (recours au privé \& } \\
\text { demandes de } \\
\text { dérogation) }\end{array}$ & 0.312 & 0.269 & 0.230 & 0.124 & 0.229 \\
\hline
\end{tabular}

On voit que, globalement, l'impact de l'évitement sur la structure sociale de la population des élèves est loin d'être négligeable. Dans quelle mesure l'évitement agit-il sur la division sociale de l'espace scolaire?

L'examen (fig.5) des coefficients de corrélation simples entre les différentes catégories d'élèves (par établissements) montre que, globalement, la structure des différenciations sociales entre les collèges se maintient : les coefficients de corrélation sont très peu affectés par l'évitement. Il est donc possible de procéder à une Analyse Factorielle des Correspondances pour repérer précisément les effets de l'évitement sur la composition sociale de chaque établissement.

Fig. 5 corrélations simples entre les différentes catégories d'élèves avant et après évitement

\begin{tabular}{|l|l|l|l|l|}
\hline Catégorie & $\mathrm{A}$ & $\mathrm{B}$ & $\mathrm{M}$ & $\mathrm{D}$ \\
\hline $\mathrm{A}(*)$ & 1 & & & \\
\hline $\mathrm{A}(* *)$ & 1 & & & \\
\hline $\mathrm{A}(* * *)$ & 1 & & & \\
\hline $\mathrm{A}(* * *))$ & 1 & & & \\
\hline $\mathrm{B}(*)$ & +0.26 & 1 & & \\
\hline $\mathrm{B}(* *)$ & +0.26 & 1 & & \\
\hline $\mathrm{B}(* * *)$ & +0.24 & 1 & & \\
\hline $\mathrm{B}(* * *))$ & +0.24 & 1 & & \\
\hline $\mathrm{M}(*)$ & -0.58 & -0.28 & 1 & \\
\hline $\mathrm{M}(* *)$ & -0.54 & -0.25 & 1 & \\
\hline $\mathrm{M}(* * *)$ & -0.55 & -0.25 & 1 & \\
\hline $\mathrm{M}(* * * *)$ & -0.50 & -0.21 & 1 & \\
\hline $\mathrm{D}(*)$ & -0.83 & -0.55 & $(-)$ & 1 \\
\hline $\mathrm{D}(* *)$ & -0.82 & -0.56 & $(-)$ & 1 \\
\hline $\mathrm{D}(* * *)$ & -0.84 & -0.55 & $(-)$ & 1 \\
\hline $\mathrm{D}(* * *))$ & -0.83 & -0.54 & $(-)$ & 1 \\
\hline
\end{tabular}

(*)Avant évitement

(**) Après recours au privé (hypothèse: pas de dérogation

(***) Après dérogations (hypothèse: pas de privé)

(****) Après évitement (compte tenu des dérogations et du recours au privé)

(-) non significatif

L'Analyse Factorielle des Correspondances en question a été réalisée sur les 106 collèges parisiens avant évitement décrits par l'effectif des quatre catégories sociales considérées. A cette structure socio-spatiale de référence, on a confronté, en les introduisant dans l'analyse sous forme «d'individus supplémentaires» trois images des 106 collèges: tels qu'ils sont après les recours au privé, tels qu'ils sont après les dérogations, tels qu'ils sont après l'évitement. On a donc en tout 106 individus statistiques plus 318 individus supplémentaires.

Le petit nombre de variables mis en œuvre et la simplicité de la structure du nuage de points font que le premier facteur rend compte de $74 \%$ de l'inertie totale, ce qui permet de le retenir 
seul. Il oppose les collèges caractérisés par la sur-représentation des élèves « favorisés $\mathrm{A}$ » (et, secondairement, «favorisés B ») aux établissements caractérisés par la sur-représentation des élèves «défavorisés » et, dans une moindre mesure, des élèves « Moyens ». Les catégories intermédiaires contribuent fort peu à la formation de l'axe, mais leur qualité de représentation n'est pas négligeable, ce qui interdit de les écarter a priori.

fig.6 Résultats de L'Analyse Factorielle des Correspondances

\begin{tabular}{|c|c|c|c|}
\hline $\begin{array}{c}\text { Variables } \\
\text { (catégorie } \\
\text { sociale) }\end{array}$ & $\begin{array}{c}\text { Coordonnée } \\
\text { sur le premier } \\
\text { facteur }\end{array}$ & $\begin{array}{c}\text { Contribution } \\
\text { au premier } \\
\text { facteur }\end{array}$ & $\begin{array}{c}\text { Qualité de } \\
\text { représentation sur } \\
\text { le premier facteur }\end{array}$ \\
\hline Favorisés A & +0.530 & 0.485 & 0.914 \\
\hline Favorisés B & +0.234 & 0.044 & 0.224 \\
\hline Moyens & -0.149 & 0.033 & 0.213 \\
\hline Défavorisés & -0.500 & 0.483 & 0.898 \\
\hline
\end{tabular}

La cartographie des résultats de cette Analyse Factorielle des Correspondances (fig.7) montre que la division sociale de l'espace scolaire parisien reste globalement la même malgré l'évitement.

-La carte de la structure sociale théorique des établissements (fig. 7a) offre l'image bien connue d'une opposition entre les quartiers bourgeois du sud-ouest et le Paris populaire du nord et de l'est. Cette structure d'ensemble bien affirmée n'exclut pas des contrastes locaux importants. Ceux-ci sont par exemple très nets dans le XVIIIe arrondissement entre les établissements de la Goutte d'Or et de la Butte Montmartre.

-La carte de la structure sociale observée dans les collèges publics après évitement (fig.7b) offre une image assez ressemblante, mais plus simple et plus contrastée. A l'échelon de Paris tout entier, les disparités socio-spatiales sont rendues plus lisibles par l'aspiration des catégories favorisées par les établissements du centre, mouvement qui aboutit d'une part à une certaine homogénéisation sociale du nord parisien, dont la singularité en termes de composition sociale apparait beaucoup plus radicale après l'évitement, et d'autre part au renforcement de la discontinuité observable entre le Paris favorisé et le Paris défavorisé (le phénomène est particulièrement net dans les IIIe et XVIIe arrondissements).

Si l'on observe l'impact de l'évitement à un échelon plus local, on constate que, malgré cette tendance générale à la simplification de la géographie sociale des établissements, les exceptions locales qui demeurent sont encore mieux différenciées de leur environnement qu'elles ne l'étaient avant évitement (premier arrondissement, porte de Saint-Cloud, Porte de Montreuil, Picpus, Ménilmontant...)

Il est donc possible de conclure que l'évitement renforce de façon significative la division sociale de l'espace parisien, aussi bien à l'échelon de l'ensemble de Paris intra-muros qu'à l'échelon local (arrondissement). Il y a toutefois des exceptions : la baisse de la position sociale d'un établissement du $6^{\mathrm{e}}$ témoigne de l'accès d'élèves défavorisés socialement, mais en situation de réussite, à un établissement prestigieux. Ceci rappelle que l'évitement, s'il compromet globalement les chances d'intégration collectives, peut être aussi, dans certaines conditions locales, un vecteur d'intégration à l'échelon de l'individu.

\section{3. évitement et ségrégation sociale}


On s'attache ici à mesurer l'impact de l'évitement sur la ségrégation sociale mesurée en termes de probabilités de contact entre élèves des différentes catégories sociales. Dans un précédent article sur la ségrégation dans l'espace scolaire francilien (François, 2002 ?), on a proposé un indice de ségrégation défini comme le rapport entre la probabilité de former un couple mixte compte-tenu de la partition en établissements et la probabilité de former un couple mixte sans tenir compte de la distribution des élèves dans les établissements. Cet indice, noté IMX, mesure en quelque sorte, dans une partition territoriale donnée, la propension à la mixité sociale: plus il est élevé, plus celle-ci est forte.

$\mathrm{IMX}=\frac{\frac{1}{E} \sum_{e=1}^{E}\left(N_{A B}^{e} / N_{P}^{e}\right)}{N_{A B} / N_{P}}$

Avec :

A et $\mathrm{B}$ deux catégories d'élèves

E le nombre d'établissements

$N_{A B} \quad$ le nombre total de couples d'élèves A,B théoriquement possibles

$N_{P} \quad$ le nombre total de couples d'élèves possibles

$N_{A B}^{e} \quad$ le nombre de couples mixtes A,B dans l'établissement e

$N_{P}^{e} \quad$ le nombre total de couples d'élèves possibles dans l'établissement e

Fig. 8 Effet de l'évitement sur la mixité sociale

\begin{tabular}{|l|l|l|l|l|}
\hline $\begin{array}{l}\text { Indice de mixité pour les } \\
\text { catégories d'élèves : }\end{array}$ & $\begin{array}{l}\text { Sans évitement (situation } \\
\text { hypothétique) }\end{array}$ & $\begin{array}{l}\text { Après les mouvements } \\
\text { vers le privé (hypothèse: } \\
\text { pas de dérogations) }\end{array}$ & $\begin{array}{l}\text { Après les mouvements de } \\
\text { dérogation } \\
\text { (hypothèse: pas de Privé) }\end{array}$ & $\begin{array}{l}\text { Avec évitement (situation } \\
\text { réelle) }\end{array}$ \\
\hline Favorisés A / Défavorisés & 0.395 & 0.338 & 0.381 & 0.369 \\
\hline Moyens / Défavorisés & 0.514 & 0.513 & 0.530 & 0.530 \\
\hline Favorisés A / Moyens & 0.455 & 0.449 & 0.445 & 0.435 \\
\hline
\end{tabular}

On voit que l'indice de mixité IMX n'est que modérément affecté par l'évitement. La probabilité de contact entre les élèves «favorisés A » d'une part, et les élèves " défavorisés » et «moyens » d'autre part passe respectivement de 0.395 à 0.369 et de 0.455 à 0.435 . On a bien une amplification de la ségrégation par l'évitement, les élèves « favorisés $A$ » se mêlant, du fait de l'évitement, moins qu'ils ne le devraient aux autres catégories habitant les mêmes quartiers, mais cette augmentation de la ségrégation reste très modeste. Entre élèves «Moyens » et «Défavorisés », en revanche, l'évitement scolaire augmente la probabilité de contact. Tout se passe donc comme si l'on tendait vers une bipolarisation de l'espace scolaire avec «favorisés» A et B d'un côté, dans des établissements attractifs et sélectifs, et «Moyens» et «Défavorisés» de l'autre, dans des établissements subis. Toutefois, la situation observée en 2001 est très loin de cette situation extrême : il faudrait pour en arriver là un volume d'évitement bien plus considérable. L'impact du recours au privé sur le même indice IMX est, quant à lui, supérieur à celui des dérogations : l'indice IMX global (recours au privé et dérogations confondus) est plus grand que l'indice IMX calculé après le recours au privé seulement. Autrement dit, le départ d'un élève "favorisé A »vers le privé augmente davantage le niveau de ségrégation que ne le fait une dérogation acceptée.

Ces résultats peuvent sembler insuffisants dans la mesure où les effets des différents mécanismes d'évitement sont confondus. Pour les distinguer, on procède à trois simulations dans lesquelles on se focalise sur les élèves «favorisés A »; ces élèves sont les plus mobiles et c'est leur mobilité qui constitue le principal enjeu de l'évitement, le jeu des établissements consistant à les attirer (ou à les retenir suivant les cas). 
Avec un premier scénario "Restons entre-nous", on cherche à évaluer l'impact de l'évitement positif des élèves « favorisés A » grâce aux dérogations (sans recours au privé). Les élèves «favorisés $\mathrm{A}$ » recherchent la proximité des élèves de la même catégorie, sans s'occuper de la présence éventuelle des élèves défavorisés. Les élèves A qui sont sectorisés dans les collèges où les élèves $\mathrm{A}$ sont proportionnellement les moins nombreux évitent ces établissements pour s'inscrire dans les collèges où les élèves A sont déjà proportionnellement les plus nombreux

Avec un deuxième scénario "Fuyons les classes populaires », on cherche à évaluer l'impact de l'évitement négatif des élèves "favorisés $\mathrm{A}$ » grâce aux dérogations (sans recours au privé). Dans ce scénario, les élèves «favorisés $A »$ fuient la proximité des élèves «défavorisés». Les élèves A qui sont sectorisés dans les collèges où les élèves «défavorisés» sont proportionnellement les plus nombreux évitent ces établissements pour s'inscrire dans les collèges où les élèves «défavorisés» sont déjà proportionnellement les moins nombreux

Avec un troisième scénario "Fuyons l'école publique », on cherche à évaluer l'impact de l'évitement négatif des élèves "favorisés A », mais , cette fois, grâce au recours au privé. Dans ce scénario, les élèves A qui sont sectorisés dans les collèges où les élèves «défavorisés» sont proportionnellement les plus nombreux évitent ces établissements pour s'inscrire dans des établissements privés.

Ces trois simulations ont pour but d'évaluer l'effet de la mobilité des élèves " favorisés A » (on suppose que les autres ne bougent pas). Elles prennent toutes pour point de départ la répartition réelle des élèves dans les collèges parisiens, et admettent les hypothèses suivantes :

1) Les parents d'élèves «favorisés A» connaissent la composition sociale des établissements.

2) Les parents d'élèves "favorisés A » choisissent l'établissement scolaire en comparant la composition sociale de leur établissement scolaire de secteur avec celle de tous les autres. La distance ou l'accessibilité ne jouent aucun rôle dans ce choix ${ }^{7}$.

3) Tous les élèves "favorisés A » situés dans un établissement concerné par l'évitement évitent et se portent vers les autres établissements (la répartition des élèves migrants se fait alors aléatoirement, proportionnellement aux effectifs des établissements visés). La catégorie d'établissements concernée par l'évitement varie selon le scénario, en outre, ses limites dépendent de l'importance globale de l'évitement que l'on choisit de simuler (soit successivement $5 \%, 25 \%, 50 \%$ puis $75 \%$ de l'effectif total des élèves A évitant).

Accepter ces hypothèses, c'est accepter de s'éloigner de la réalité des pratiques d'évitement : il est très improbable par exemple que tous les élèves «favorisés A » sans exception évitent un établissement. L'intérêt de la simulation réside donc moins dans la comparaison avec les situations observées que dans la comparaison des scénarii entre eux pour démêler quelles pratiques induisent, toutes choses égales par ailleurs, le plus de ségrégation.

Fig.9 Effet de l'évitement sur la ségrégation mesurée par l'indice IMX (simulations)

\begin{tabular}{|l|l|l|l|l|}
\hline Proportion d'élèves « favorisés A » évitant & $5 \%$ & $25 \%$ & $50 \%$ & $75 \%$ \\
\hline Scénario1 « Restons entre-nous » & 0.351 & 0.239 & 0.108 & 0.029 \\
\hline
\end{tabular}

\footnotetext{
${ }^{7}$ Ceci n'est pas trop éloigné de la réalité : on verra plus loin que les élèves « favorisés A » qui évitent ne font, en effet, pas grand cas de la distance.
} 


\begin{tabular}{|l|l|l|l|l|}
\hline Evitement positif des élèves « favorisés A » (pas de recours au privé) & & & & \\
\hline $\begin{array}{l}\text { Scénario2 « Fuyons les classes populaires » (pas de recours au privé) } \\
\text { Evitement négatif des élèves « favorisés A » }\end{array}$ & 0.365 & 0.319 & 0.260 & 0.207 \\
\hline $\begin{array}{l}\text { Scénario 3 « Fuyons l'école publique » } \\
\text { Evitement négatif des élèves « favorisés A » exclusivement par le recours au privé }\end{array}$ & 0.361 & 0.273 & 0.180 & 0.102 \\
\hline
\end{tabular}

Dans la configuration de l'espace scolaire parisien, l'évitement positif est plus ségrégatiogène que l'évitement négatif. En effet, seul le premier scénario (évitement positif) permet d'envisager, avec un indice proche de zéro, une situation de ségrégation quasi absolue dans le cas d'un évitement effectué par les trois quarts des élèves «favorisés A » (la totalité des élèves "favorisés $A$ » se trouve alors concentrée dans une quinzaine d'établissements peuplés presque exclusivement de ces élèves.). Avec la même proportion d'élèves évitants, la probabilité de contact entre les élèves «favorisés A » et les autres s'élève encore à plus de $20 \%$ du maximum théorique dans le deuxième scénario (évitement négatif).

En matière d'évitement négatif, le recours au privé génère nettement plus de ségrégation que l'évitement par la dérogation. La comparaison des résultats des deux derniers scénarii montre que l'écart de ségrégation induit augmente avec la proportion d'élèves «Favorisés A» que l'on voit éviter, jusqu'à varier du simple au double (pour trois quart d'élèves A évitant). Autrement dit, plus l'évitement est massif, plus le recours au privé est un facteur aggravant de la ségrégation. Or, non seulement l'évitement réel s'accroît en île de France d'année en année, mais encore la proportion d'élèves recourrant au secteur privé à l'entrée en sixième augmente régulièrement.

Toutefois le recours au privé joue dans la ségrégation scolaire un rôle ambivalent. En effet, si l'on observe ce qui se passerait en l'absence de toute référence au contexte social de l'aire de recrutement, on obtient le résultat suivant :

Si 50\% des élèves «favorisés A », choisis au hasard dans tous les établissements (sans effet de contexte local, donc) allaient dans le privé, l'indice IMX augmenterait (de 0.395 à 0.415). Ceci peut s'expliquer de la façon suivante:

Les établissements des Beaux Quartiers sont très spécialisés en élèves «favorisés A » (la proportion maximale d'élèves A observée avant évitement est de 68\%); après le départ d'une partie des élèves A vers le privé ils deviennent, par construction, nettement moins spécialisés, leur profil social est plus équilibré, ce qui influence notablement l'indice. Dans la pratique, ce résultat théorique est illustré de la façon suivante: on peut observer que, paradoxalement, le choix du privé par une partie des familles dans les Beaux quartiers libère un certain nombre de places et permet, via la dérogation, à un public socialement plus diversifié que la population résidente d'accéder à des établissements prestigieux (ce qui se produit par exemple dans le $\mathrm{VI}^{\mathrm{e}}$ arrondissement).

De tels effets demeurent évidemment marginaux. Il permettent toutefois de rappeler que ce sont les facteurs locaux qui sont les plus importants: le départ de tous les élèves A d'un établissement où ils sont déjà très peu nombreux aura une répercussion relativement faible sur l'indice IMX global mais des conséquences très importantes sur l'établissement, et, par ricochet, sur l'ensemble du système. On a vu que l'écrémage par évitement vers le privé est particulièrement fort dans les établissements qui sont déjà les plus défavorisés de par la 
composition de la population résidente de leur secteur de recrutement ; l'évitement est alors particulièrement important parmi les catégories moyennes. Cette prise en compte du contexte dans la décision est confirmée d'une part lors des entretiens avec les parents d'élèves dérogeant et d'autre part dans des travaux antérieurs utilisant les dossiers individuels de dérogation, là où le formulaire de dérogation est ainsi conçu que le motif choisi par la famille est libellé librement (François, 1995).

Dans ces simulations de l'indice de ségrégation IMX, on a postulé que la structure sociale des établissements était la cause essentielle de l'évitement. En revenant à l'observation des mouvements réels des élèves, on va tenter à présent de démêler quelle est dans les phénomènes d'évitement l'importance relative de cette structure sociale, de l'évolution de celle-ci ensuite et du contexte géographique enfin.

\section{Les causes de l'évitement}

Pour prétendre démêler les causes de l'évitement, il était nécessaire de neutraliser l'effet de structure. En effet, la propension à éviter des différentes catégories étant très inégales, l'intensité locale de l'évitement dépend «mécaniquement» de la composition sociale. Pour neutraliser cet artefact, différentes solutions sont possibles. Dans la mesure où il n'est pas certain, a priori, que les stratégies d'évitement des différentes catégories d'élèves relèvent des mêmes logiques, tenter d'expliquer séparément l'évitement de chacune des catégories d'élèves a paru la meilleure solution.

\section{1. une cause structurelle possible : le contexte social}

Fig.10 matrice des corrélations simples entre propensions à éviter et structure sociale des aires de recrutement.

\begin{tabular}{|c|c|c|c|c|c|}
\hline & $\begin{array}{l}\text { Coordonnée } \\
\text { sur le premier } \\
\text { axe de l'AFC }\end{array}$ & $\begin{array}{l}\text { Proportion } \\
\text { d'élèves } \\
\text { "favorisés A" }\end{array}$ & $\begin{array}{l}\text { Proportion } \\
\text { d'élèves } \\
\text { "favorisés B" }\end{array}$ & $\begin{array}{l}\text { Proportion } \\
\text { d'élèves } \\
\text { "moyens" }\end{array}$ & $\begin{array}{l}\text { Proportion } \\
\text { d'élèves } \\
\text { "défavorisés" }\end{array}$ \\
\hline Propension à déroger (catégorie $\mathrm{A}$ ) & -0.39 & -0.41 & $*$ & $*$ & +0.36 \\
\hline $\begin{array}{l}\text { Propension à recourir au privé } \\
\text { (catégorie A) }\end{array}$ & -0.38 & -0.43 & $*$ & +0.24 & +0.31 \\
\hline Propension à éviter (catégorie A) & -0.48 & -0.52 & $*$ & +0.24 & +0.43 \\
\hline Propension à déroger (catégorie B) & -0.22 & -0.28 & $*$ & $*$ & $*$ \\
\hline $\begin{array}{l}\text { Propension à recourir au privé } \\
\text { (catégorie B) }\end{array}$ & -0.34 & -0.32 & $*$ & $*$ & +0.33 \\
\hline Propension à éviter (catégorie B) & -0.32 & -0.36 & $*$ & $*$ & +0.30 \\
\hline $\begin{array}{l}\text { Propension à déroger (catégorie } \\
\text { «Moyens ») }\end{array}$ & -0.43 & -0.46 & $*$ & +0.34 & +0.34 \\
\hline $\begin{array}{l}\text { Propension à recourir au privé } \\
\text { (catégorie « Moyens ») }\end{array}$ & -0.28 & -0.27 & $*$ & $*$ & +0.30 \\
\hline $\begin{array}{l}\begin{array}{l}\text { Propension à éviter (catégorie } \\
\text { «Moyens ») }\end{array} \\
\end{array}$ & -0.46 & -0.49 & $*$ & +0.25 & +0.41 \\
\hline $\begin{array}{l}\text { Propension à déroger (catégorie } \\
\text { "défavorisés") }\end{array}$ & -0.30 & -0.30 & $*$ & $*$ & +0.29 \\
\hline $\begin{array}{l}\text { Propension à recourir au privé } \\
\text { (catégorie "défavorisés") }\end{array}$ & -0.28 & -0.28 & $*$ & $*$ & +0.28 \\
\hline $\begin{array}{l}\text { Propension à éviter (catégorie } \\
\text { "défavorisés") }\end{array}$ & -0.36 & -0.35 & $*$ & $*$ & +0.34 \\
\hline
\end{tabular}

Les corrélations non significatives au seuil de $5 \%$ sont indiquées par $\left.{ }^{*}\right)$ 
La coordonnée sur le premier axe de l'AFC, qui résume bien la structure sociale des établissements, est corrélée négativement avec la propension à éviter de chacune des catégories. Comme on pouvait s'y attendre, il y a donc un lien significatif entre la composition sociale et l'importance de l'évitement. Plus la position de l'établissement dans l'échelle sociale est basse (coordonnée très négative sur l'axe 1), plus l'évitement est important, et réciproquement. Si l'on veut préciser ce résultat, on voit qu'une surreprésentation d'élèves «favorisés A » est associée de façon significative à un évitement modéré pour toutes les catégories d'élèves. Symétriquement, la proportion d'élèves « défavorisés » semble agir comme un aiguillon encourageant l'évitement (sauf en ce qui concerne la propension à déroger des élèves «favorisés B » pour lesquels la relation n'est pas significative). L'importance des catégories intermédiaires apparaît beaucoup moins déterminante: le pouvoir explicatif de la proportion d'élèves «favorisés B » n'est significatif dans aucun cas; la proportion d'élèves «moyens » semble encourager à éviter, mais elle ne joue de façon significative que dans le cas des élèves «favorisés A » et des élèves «moyens » eux-mêmes. Faut-il y voir un effet de l'hétérogénéité de cette dernière catégorie?

On voit que, s'il existe bien une relation globalement significative entre la composition sociale d'un secteur de recrutement et l'évitement de l'établissement correspondant, l'intensité de cette relation est, dans tous les cas, relativement faible (le coefficient de détermination le plus élevé ne dépasse pas $25 \%$ ). Il est donc possible de considérer un autre facteur susceptible d'expliquer la propension à l'évitement: l'évolution récente de la structure sociale des établissements.

\section{2. l'évolution de la structure sociale des établissements influence-t-elle la propension à éviter?}

On pourrait penser que l'évitement est motivé par une mauvaise image de l'établissement, et que celle-ci va de paire avec une baisse locale du niveau social ; or, c'est exactement l'inverse qui se produit à Paris intra-muros (fig.11). Si l'évitement des catégories moyennes et défavorisées n'a pas de relation significative avec l'évolution sociale du secteur de recrutement, l'évitement des catégories supérieures est corrélé positivement avec la hausse des collèges dans la hiérarchie sociale.

Fig. 11 structure sociale et propensions à éviter (corrélations simples)

\begin{tabular}{|l|l|l|}
\hline & $\begin{array}{l}\text { Coordon } \\
\text { née sur } \\
\text { le } \\
\text { premier } \\
\text { axe de } \\
\text { l'AFC } \\
\text { coordonnée entre } \\
\text { (rappel) }\end{array}$ & \\
\hline Propension à déroger (catégorie A) & -0.39 & +0.32 \\
\hline Propension à recourir au privé (catégorie A) & -0.38 & $(*)$ \\
\hline Propension à éviter (catégorie A) & -0.48 & +0.34 \\
\hline Propension à déroger (catégorie B) & -0.22 & +0.40 \\
\hline Propension à recourir au privé (catégorie B) & -0.34 & $(*)$ \\
\hline Propension à éviter (catégorie B) & -0.32 & +0.41 \\
\hline Propension à déroger (catégorie M) & -0.43 & $(*)$ \\
\hline
\end{tabular}




\begin{tabular}{|l|l|l|}
\hline Propension à recourir au privé (catégorie M) & -0.28 & $\left(^{*}\right)$ \\
\hline Propension à éviter (catégorie M) & -0.46 & $\left(^{*}\right)$ \\
\hline Propension à déroger (catégorie D) & -0.30 & $\left(^{*}\right)$ \\
\hline Propension à recourir au privé (catégorie D) & -0.28 & $\left(^{*}\right)$ \\
\hline Propension à éviter (catégorie D) & -0.36 & $\left(^{*}\right)$ \\
\hline
\end{tabular}

(*) coefficient non significatif au seuil de $5 \%$

Ce résultat paradoxal trouve son explication dans les dynamiques de recomposition sociale à l'œuvre dans l'agglomération parisienne. L'embourgeoisement de nombreux quartiers parisiens anciennement populaires introduit un hiatus toujours croissant entre la composition sociale de la population résidente et celle de la population des élèves des collèges. Espace résidentiel et espace scolaire sont de plus en plus déconnectés par le jeu des pratiques d'évitement. Un ménage socialement favorisé peut très bien, en ce qui concerne son choix résidentiel, s'accommoder d'un quartier encore populaire et abritant de nombreux étrangers (cette proximité est même souvent recherchée, comme en attestent les témoignages récoltés auprès des parents d'élèves «c'est un quartier animé, pittoresque, etc.. »), mais de là à y scolariser sa progéniture, il y a un pas que beaucoup hésitent à franchir. Cette tendance, qui apparaît ici nettement, est probablement sous-estimée par les mesures de l'évitement dont on a pu disposer. Pour les ménages venus récemment d'un autre quartier pour trouver un logement (un peu) moins cher, la tentation est forte en effet de continuer à scolariser leurs enfants dans leur quartier d'origine: il s'agit alors généralement d'une forme illicite d'évitement qui n'est pas prise en compte ici.

Les facteurs explicatifs introduits jusqu'ici ne tiennent compte que de facteurs sociologiques, sans égard pour la situation géographique des établissements. Celle-ci est-elle un facteur explicatif recevable?

\subsection{Contexte géographique et évitement}

On souhaite vérifier dans quelle mesure le contexte géographique des aires de recrutement est susceptible d'influencer l'évitement. Pour rendre compte d'un éventuel «effet d'appel» de l'offre scolaire locale, on a tout d'abord calculé les corrélations simples (fig.12) entre les variables décrivant l'évitement d'une part, et les différences de structure sociale entre l'établissement évité et ses voisins d'autre part. Le «voisinage » retenu ici est constitué des aires de recrutement immédiatement contiguës à celle de l'établissement évité. La structure sociale de ce voisinage est décrite successivement par la proportion d'élèves "favorisés A », la proportion d'élèves «favorisés B », la coordonnée sur le premier facteur de l'AFC. Pour chacune de ces variables, on a encore retenu trois façons d'appréhender l'écart entre l'établissement évité et son voisinage : on a mesuré successivement la différence entre la valeur observée pour l'établissement et -la moyenne du voisinage, -le minimum observé dans le voisinage, -le maximum observé dans le voisinage, en rapportant à chaque fois le résultat à la moyenne du voisinage ${ }^{8}$.

fig.12 évitement et structure sociale du voisinage (corrélations simples)

\begin{tabular}{|c|c|c|c|c|c|c|c|c|c|}
\hline 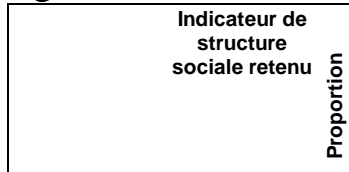 & 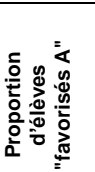 & 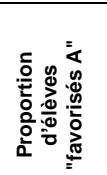 & 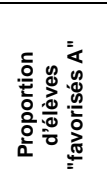 & 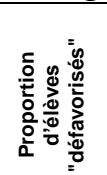 & 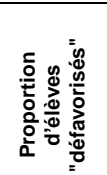 & 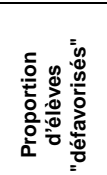 & 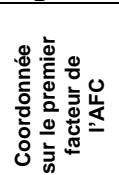 & 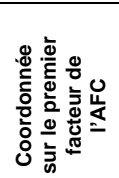 & 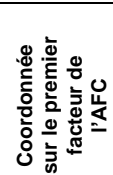 \\
\hline
\end{tabular}

\footnotetext{
${ }^{8} \mathrm{D}$ 'autres indicateurs ont été essayés, mais leur relation statistique avec l'évitement étant nettement plus faible, ils n'ont pas été retenus.
} 


\begin{tabular}{|c|c|c|c|c|c|c|c|c|c|c|}
\hline & $\begin{array}{c}\text { Mesure de la } \\
\text { différence avec } \\
\text { la structure } \\
\text { sociale } \\
\text { observée dans } \\
\text { le voisinage }\end{array}$ & 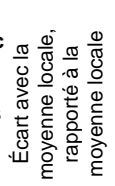 & 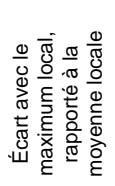 & 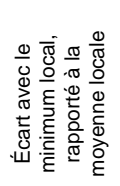 & 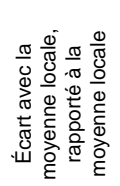 & 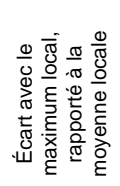 & 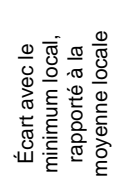 & 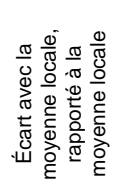 & 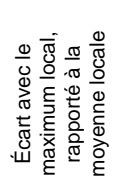 & 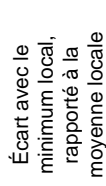 \\
\hline \multirow[t]{4}{*}{\begin{tabular}{|l}
$\begin{array}{l}\text { propension à } \\
\text { déroger... }\end{array}$ \\
\end{tabular}} & $\begin{array}{l}\text { parmi les élèves } \\
\text { "favorisés A" }\end{array}$ & -0.28 & -0.38 & $\left(^{*}\right)$ & $\left({ }^{*}\right)$ & $\left(^{*}\right)$ & $\left({ }^{*}\right)$ & -0.27 & -0.27 & -0.23 \\
\hline & $\begin{array}{l}\text { parmi les élèves } \\
\text { "favorisés B" } \\
\text { parmi les élèves }\end{array}$ & $\left({ }^{\star}\right)$ & -0.30 & $\left({ }^{*}\right)$ & $\left(^{*}\right)$ & $\left({ }^{*}\right)$ & $\left({ }^{*}\right)$ & $\left({ }^{*}\right)$ & -0.26 & $\left({ }^{*}\right)$ \\
\hline & "moyens" & -0.30 & -0.33 & $\left({ }^{*}\right)$ & $\left({ }^{*}\right)$ & $\left({ }^{*}\right)$ & $\left({ }^{*}\right)$ & -0.29 & -0.30 & -0.26 \\
\hline & $\begin{array}{l}\text { parmi les élèves } \\
\text { "défavorisés" }\end{array}$ & -0.22 & -0.32 & $\left.{ }^{*}\right)$ & $\left({ }^{*}\right)$ & $\left.{ }^{*}\right)$ & $\left({ }^{*}\right)$ & -0.24 & -0.34 & -0.19 \\
\hline \multirow[t]{5}{*}{$\begin{array}{l}\text { recours au } \\
\text { privé ... }\end{array}$} & $\begin{array}{l}\text { parmi les élèves } \\
\text { "favorisés A" }\end{array}$ & -0.34 & -0.39 & -0.30 & $\left(^{*}\right)$ & $\left(^{*}\right)$ & $\left({ }^{\star}\right)$ & -0.32 & -0.34 & -0.34 \\
\hline & $\begin{array}{l}\text { parmi les élèves } \\
\text { "favorisés B" }\end{array}$ & -0.33 & -0.42 & -0.23 & -0.23 & -0.22 & $\left({ }^{*}\right)$ & -0.44 & -0.53 & -0.36 \\
\hline & parmi les élèves & & & & & & & & & \\
\hline & "moyens" & -0.28 & -0.34 & $\left({ }^{*}\right)$ & -0.21 & $\left({ }^{*}\right)$ & $\left({ }^{*}\right)$ & $\left({ }^{*}\right)$ & -0.26 & -0.20 \\
\hline & $\begin{array}{l}\text { parmi les élèves } \\
\text { "défavorisés" }\end{array}$ & $\left({ }^{*}\right)$ & -0.26 & $\left.{ }^{*}\right)$ & $\left({ }^{*}\right)$ & $\left.{ }^{*}\right)$ & $\left({ }^{*}\right)$ & $\left.{ }^{*}\right)$ & $\left(^{*}\right)$ & $\left({ }^{*}\right)$ \\
\hline \multirow[t]{5}{*}{$\begin{array}{l}\text { propension à } \\
\text { éviter ... }\end{array}$} & $\begin{array}{l}\text { parmi les élèves } \\
\text { "favorisés A" }\end{array}$ & -0.37 & -0.47 & -0.23 & $\left(^{*}\right)$ & $\left({ }^{\star}\right)$ & $\left(^{*}\right)$ & -0.36 & -0.37 & -0.34 \\
\hline & $\begin{array}{l}\text { parmi les élèves } \\
\text { "favorisés B" }\end{array}$ & -0.25 & -0.41 & $\left({ }^{*}\right)$ & $\left({ }^{*}\right)$ & $(*)$ & $(*)$ & -0.27 & -0.41 & -0.20 \\
\hline & parmi les élèves & & & & & & & & & \\
\hline & "moyens" & -0.34 & -0.41 & -0.20 & -0.21 & $\left({ }^{*}\right)$ & $\left({ }^{*}\right)$ & -0.31 & -0.35 & -0.29 \\
\hline & $\begin{array}{l}\text { parmi les élèves } \\
\text { "défavorisés" }\end{array}$ & -0.24 & -0.37 & $\left(^{*}\right)$ & $\left({ }^{*}\right)$ & $\left({ }^{*}\right)$ & $\left({ }^{*}\right)$ & -0.25 & -0.37 & -0.20 \\
\hline
\end{tabular}

On voit que s'il est souvent possible d'établir une relation statistique significative entre l'évitement d'un établissement et la structure sociale des établissements voisins, cette relation, toujours inverse, demeure généralement modeste (le coefficient de corrélation simple n'excède jamais -0.5). Les proportions d'élèves « défavorisés » observées dans le voisinage entretiennent peu de relations avec l'évitement. En effet, les seuls coefficients significatifs relevés sont bien modestes. Ils ne concernent que les catégories intermédiaires (élèves «favorisés B » et «moyens »), et seulement lorsque l'on tient compte du recours au secteur privé. Il s'agit ici d'évitement négatif: quand l'environnement n'est pas satisfaisant, on se tourne vers le privé.

A l'opposé, l'indicateur le plus pertinent pour expliquer l'évitement est la proportion d'élèves "favorisés A » dans le voisinage. La proportion maximum d'élèves "favorisés A », en particulier, est l'indicateur le mieux corrélé avec l'évitement dans tous les cas (sauf celui de la propension à recourir au privé des élèves B). Tout se passe comme si l'environnement géographique jouait un rôle plus important dans l'évitement positif que dans l'évitement négatif: dans le cas général, on évite quand il y a un "meilleur choix » à proximité, ce «meilleur choix» perçu coïncidant probablement assez bien avec l'établissement du voisinage dont la proportion d'élèves « favorisés A » est la plus forte - ou, plus généralement, dont la position dans l'échelle sociale est la plus élevée (coordonnée sur le premier facteur de l'AFC).

Toutefois, il convient de rester prudent dans l'interprétation de ces résultats, dans la mesure l'évitement $n$ 'est pas distribué au hasard: la géographie de l'évitement recoupe en partie celle de la composition sociale des établissements. On voit qu'il est nécessaire d'isoler ce qui, dans l'évitement observé, peut relever en propre d'un effet du voisinage, et ce qui est dû à l'effet de structure observé plus haut. A cet effet, on procède à une régression multiple «pas à pas » pour laquelle on reprend les facteurs explicatifs dont on a déjà vu qu'ils jouaient, individuellement, un rôle significatif. 
Fig. 13 parts respectives de la structure sociale, de l'évolution de celle-ci et du voisinage dans l'explication de l'évitement (régression multiple)

\begin{tabular}{|c|c|c|c|c|c|c|c|}
\hline & & $\begin{array}{l}\text { 1ere ligne : } \\
\text { constante } \\
2^{\mathrm{e}} \text { ligne : part de } \\
\text { variance } \\
\text { expliquée par le } \\
\text { modèle }\end{array}$ & $\begin{array}{l}\text { Variable } \\
\text { explicative } 1 \\
\text { Proportion } \\
\text { d'élèves } \\
\text { "favorisés } \\
\text { A " }\end{array}$ & $\begin{array}{l}\text { Variable } \\
\text { explicative 2 } \\
\text { Proportion } \\
\text { d'élèves } \\
\text { "défavorisés" }\end{array}$ & $\begin{array}{l}\text { Variable } \\
\text { explicative } 3 \\
\text { Evolution de la } \\
\text { position sociale } \\
\text { entre } 1997 \text { et } \\
2001\end{array}$ & $\begin{array}{l}\text { Variable explicative } 4 \\
\text { Écart avec le maximum } \\
\text { local, rapporté à la } \\
\text { moyenne locale } \\
\text { (élèves " favorisés A ") }\end{array}$ & $\begin{array}{l}\text { Variable explicative } 5 \\
\text { mÉcart absolu avec le } \\
\text { maximum local (position } \\
\text { sociale mesuree par la } \\
\text { ")coordonné sur le premier } \\
\text { facteur de l'AFC) }\end{array}$ \\
\hline $\begin{array}{l}\text { PROPderA } \\
\text { propension à } \\
\text { déroger des élèves } \\
\text { «Favorisés A » } \\
\end{array}$ & $\begin{array}{l}\text { Coefficient } \\
\text { Part de } \\
\text { variance } \\
\text { prise en } \\
\text { compte } \\
\end{array}$ & $\begin{array}{l}0.284 \\
0.264\end{array}$ & $\begin{array}{l}-0.551 \\
\mathbf{0 . 1 7 0}\end{array}$ & $\left({ }^{*}\right)$ & $\begin{array}{l}0.314 \\
0.094\end{array}$ & $\left({ }^{*}\right)$ & $\left({ }^{*}\right)$ \\
\hline $\begin{array}{l}\text { PROPderB } \\
\text { propension à } \\
\text { déroger des élèves } \\
\text { «Favorisés B » }\end{array}$ & \begin{tabular}{|l} 
Coefficient \\
Part de \\
variance \\
prise en \\
compte \\
\end{tabular} & 0.193 & -0.441 & $\left({ }^{*}\right)$ & 0.472 & $\left({ }^{*}\right)$ & $\left({ }^{*}\right)$ \\
\hline \begin{tabular}{|l|} 
PROPderM \\
propension à \\
déroger des élèves \\
« moyens » \\
\end{tabular} & \begin{tabular}{|l} 
Coefficient \\
Part de \\
variance \\
prise en \\
compte \\
\end{tabular} & $\begin{array}{l}0.137 \\
0.209\end{array}$ & $\begin{array}{l}-0.419 \\
\mathbf{0 . 2 0 9}\end{array}$ & $\left({ }^{*}\right)$ & $\left({ }^{*}\right)$ & $\left({ }^{*}\right)$ & $\left({ }^{*}\right)$ \\
\hline \begin{tabular}{|l|} 
PROPderD \\
propension à \\
déroger des élèves \\
«défavorisés » \\
\end{tabular} & \begin{tabular}{|l} 
coefficient \\
Part de \\
variance \\
prise en \\
compte \\
\end{tabular} & $\begin{array}{l}-0.052 \\
0.116\end{array}$ & & $\left({ }^{*}\right)$ & $\left({ }^{*}\right)$ & $\left({ }^{*}\right)$ & $\begin{array}{l}-0.223 \\
\mathbf{0 . 1 1 6}\end{array}$ \\
\hline \begin{tabular}{|l} 
PROPpriA \\
propension à \\
recourir au privé des \\
élèves «Favorisés \\
A »
\end{tabular} & \begin{tabular}{|l} 
Coefficient \\
Part de \\
variance \\
prise en \\
compte \\
\end{tabular} & $\begin{array}{l}0.203 \\
0.184\end{array}$ & $\begin{array}{l}-0.287 \\
\mathbf{0 . 1 8 4}\end{array}$ & $\left({ }^{*}\right)$ & $\left({ }^{*}\right)$ & $\left({ }^{*}\right)$ & $\left({ }^{*}\right)$ \\
\hline \begin{tabular}{|l} 
PROPpriB \\
propension à \\
recourir au privé des \\
élèves « Favorisés \\
B »
\end{tabular} & \begin{tabular}{|l} 
coefficient \\
Part de \\
variance \\
prise en \\
compte \\
\end{tabular} & $\begin{array}{l}0.030 \\
0.276\end{array}$ & $\left({ }^{*}\right)$ & $\left({ }^{*}\right)$ & $\left({ }^{*}\right)$ & $\begin{array}{l}-0.210 \\
\mathbf{0 . 2 7 6}\end{array}$ & $\left({ }^{*}\right)$ \\
\hline $\begin{array}{l}\text { PROPpriM } \\
\text { propension à } \\
\text { recourir au privé des } \\
\text { élèves «Moyens» } \\
\end{array}$ & \begin{tabular}{|l} 
Coefficient \\
Part de \\
variance \\
prise en \\
compte \\
\end{tabular} & $\begin{array}{l}0.044 \\
0.116\end{array}$ & $\left({ }^{*}\right)$ & $\left({ }^{*}\right)$ & $\left({ }^{*}\right)$ & $\begin{array}{l}-0.055 \\
\mathbf{0 . 1 1 6}\end{array}$ & $\left({ }^{*}\right)$ \\
\hline $\begin{array}{l}\text { PROPpriD } \\
\text { propension à } \\
\text { recourir au privé des } \\
\text { élèves } \\
\text { « Défavorisés» } \\
\end{array}$ & \begin{tabular}{|l} 
Coefficient \\
Part de \\
variance \\
prise en \\
compte \\
\end{tabular} & $\begin{array}{l}0.000 \\
0.140\end{array}$ & $\left({ }^{*}\right)$ & $\begin{array}{l}0.074 \\
0.081\end{array}$ & $\begin{array}{l}0.043 \\
0.059\end{array}$ & $\left({ }^{*}\right)$ & $\left({ }^{*}\right)$ \\
\hline $\begin{array}{l}\text { PROPeviA } \\
\text { propension à éviter } \\
\text { des élèves } \\
\text { «Favorisés A } \\
\end{array}$ & $\begin{array}{l}\text { Coefficient } \\
\text { Part de } \\
\text { variance } \\
\text { prise en } \\
\text { compte } \\
\end{array}$ & $\begin{array}{l}0.492 \\
0.375\end{array}$ & $\begin{array}{l}-0.834 \\
\mathbf{0 . 2 7 2}\end{array}$ & $\left({ }^{*}\right)$ & $\begin{array}{l}0.393 \\
0.103\end{array}$ & $\left({ }^{*}\right)$ & $\left({ }^{*}\right)$ \\
\hline $\begin{array}{l}\text { PROPeviB } \\
\text { propension à éviter } \\
\text { des élèves } \\
\text { «Favorisés B » } \\
\end{array}$ & \begin{tabular}{|l} 
Coefficient \\
Part de \\
variance \\
prise en \\
compte \\
\end{tabular} & $\begin{array}{l}0.026 \\
0.320\end{array}$ & $\left({ }^{*}\right)$ & $\left({ }^{*}\right)$ & $\begin{array}{l}0.538 \\
0.149\end{array}$ & $\left({ }^{*}\right)$ & $\begin{array}{l}-0.445 \\
\mathbf{0 . 1 7 0}\end{array}$ \\
\hline $\begin{array}{l}\text { PROPeviM } \\
\text { propension à éviter } \\
\text { des élèves } \\
\text { « Moyens» } \\
\end{array}$ & \begin{tabular}{|l} 
Coefficient \\
Part de \\
variance \\
prise en \\
compte \\
\end{tabular} & $\begin{array}{l}0.251 \\
0.241\end{array}$ & $\begin{array}{l}-0.557 \\
\mathbf{0 . 2 4 1}\end{array}$ & $\left({ }^{*}\right)$ & $\left({ }^{*}\right)$ & $\left({ }^{*}\right)$ & $\left({ }^{*}\right)$ \\
\hline PROPeviD & coefficient & -0.033 & $\left({ }^{*}\right)$ & $\left({ }^{*}\right)$ & $\left({ }^{*}\right)$ & $\left({ }^{*}\right)$ & -0.128 \\
\hline
\end{tabular}


\begin{tabular}{|l|lll|}
$\begin{array}{l}\text { propension à éviter } \\
\text { des élèves } \\
\text { « Défavorisés» }\end{array}$ & $\begin{array}{l}\text { Part de } \\
\text { variance } \\
\text { prise en } \\
\text { compte }\end{array}$ & $\mathbf{0 . 1 3 6}$ & 0.136 \\
\hline$\left(^{*}\right)$ relation non significative
\end{tabular}

$\mathrm{Nb}$ : on n'a reporté dans ce tableau que les variables explicatives qui présentaient au moins un coefficient significatif.

La première remarque qui s'impose concerne la faiblesse de la part de variance expliquée par les différents modèles. Des essais avec d'autres variables ou un voisinage plus large n'ont pas donné de meilleurs résultats. La prise en compte de la structure sociale de l'espace résidentiel n'apporte aucune amélioration, cette variable étant liée à la structure sociale de l'établissement. On doit donc constater que la structure sociale d'un établissement et de son voisinage, s'ils sont des facteurs explicatifs recevables, ne sauraient suffire à eux seuls à expliquer l'évitement. On peut penser que d'autres motifs ${ }^{9}$, qu'il n'est pas toujours facile de saisir à travers des indicateurs statistiques, sont susceptibles de jouer un rôle déterminant dans l'évitement. Il faut également rappeler qu'en matière d'évitement, les choix des familles sont déterminés moins par les divisions sociales réelles de l'espace scolaire que par les représentations qu'elles se font de celles-ci. L'écart entre représentations et réalités reste à étudier, et il y a fort à parier qu'il varie beaucoup au sein de l'espace scolaire.

Cependant, pour modestes qu'elles soient, les relations mises en évidence par la régression multiple n'en sont pas moins significatives.

Le rôle des variables explicatives qui rendent compte de la structure sociale est prépondérant pour les élèves favorisés A ( $27 \%$ expliqués pour la propension à éviter) mais aussi pour les élèves «moyens ». En ce qui concerne les élèves «défavorisés, la structure sociale joue différemment. Alors que partout ailleurs la structure sociale joue par l'intermédiaire de la proportion d'élèves favorisés ou de la position sociale globale de l'établissement, c'est la proportion d'élèves défavorisés qui remplit ce rôle quand il s'agit d'expliquer le recours au privé des élèves défavorisés. Plus la proportion d'élèves défavorisés est importante dans un établissement, plus ceux-ci ont tendance à se tourner vers le secteur privé.

L'évolution de la structure sociale (embourgeoisement de l'aire de recrutement) joue un rôle non négligeable dans l'évitement des élèves favorisés (surtout les élèves « favorisés B » pour l'évitement desquels c'est le premier facteur explicatif). Le comportement des élèves défavorisés, lui aussi, est affecté par l'évolution de la composition sociale de l'établissement : la propension de ces derniers à recourir au privé est en effet liée, modestement mais significativement, à l'amélioration de la position sociale de l'établissement. Une hypothèse vraisemblable est celle d'une diffusion sociale des pratiques d'évitement au sein des établissements, les élèves défavorisés adoptant les pratiques d'évitement des autres catégories.

Le voisinage n'est un facteur explicatif significatif que pour les catégories intermédiaires (élèves «favorisés B » et «moyens ) et défavorisées. C'est le principal facteur permettant d'expliquer propension à déroger et propension à recourir au privé des élèves défavorisés, et, surtout, la propension à éviter des catégories intermédiaires $(28 \%$ de la variance de la propension à recourir au privé des élèves moyens est expliquée par le voisinage). Le rôle du voisinage géographique dans l'évitement géographique est socialement différencié : pour les élèves les plus favorisés, il est impossible d'établir un lien significatif : c'est la structure sociale de l'établissement évité qui compte avant tout. Si celle-ci n'est pas satisfaisante,

\footnotetext{
${ }^{9}$ L'origine nationale des élèves évitants et évité, qui n'a pu être intégrée dans cet article faute de place, est au centre d'une recherche qui viendra compléter ces résultats.
} 
l'élève favorisé évite souvent, mais sans égards pour l'offre scolaire du voisinage. Il est suffisamment informé et suffisamment mobile pour faire son choix parmi l'ensemble des établissements parisiens, n'hésitant pas à franchir des distances parfois très importantes. En revanche, les autres catégories d'élèves semblent davantage influencées par l'offre scolaire du voisinage : moins les élèves sont favorisés socialement, plus leur marge de manœuvre est étroite. Les élèves défavorisés, on l'a vu, évitent moins que les autres ; quand ils le font, c'est à courte portée. On a même vu des familles modestes protester contre une modification de la carte scolaire qui leur donnait accès à un établissement recherché, mais plus éloigné de leur domicile: les coûts du trajet leur paraissaient prohibitifs.

\section{Le système de l'écrémage}

On a pu constater l'importance que peut prendre localement l'évitement sélectif appelé "écrémage" par les acteurs. La composition sociale de la population des élèves d'un collège ne peut à elle seule suffire à expliquer le mécanisme de cet écrémage. On a vu l'importance de la diversité locale de l'offre scolaire, et celle d'un éventuel processus d'embourgeoisement à l'oeuvre dans le quartier. Ces différents éléments peuvent être mobilisés pour proposer une schématisation du système de l'écrémage valable pour les collèges où la proportion d'élèves d'origine défavorisée est assez forte (Fig. 14). Au centre du système on trouve en effet une forte proportion d'élèves défavorisés qui conduit certains élèves à pratiquer l'évitement. Ces derniers sont plus nombreux parmi les élèves d'origine favorisée en situation de réussite scolaire. Les pratiques d'évitement renforcent à leur tour, par une classique boucle de rétroaction positive, la sur-représentation des élèves d'origine défavorisée... Mais la fréquence des pratiques d'évitement interagit également avec l'hétérogénéité locale. D'une part, une hétérogénéité spatiale à l'intérieur de l'aire de recrutement du collège, en particulier si, spatialement organisée, elle se traduit par une discontinuité, encourage l'évitement du collège par les élèves résidant dans le sous-espace le moins défavorisé. D'autre part, une forte hétérogénéité spatiale dans le voisinage de l'aire de recrutement du collège considéré se traduit par une offre scolaire plus diversifiée et influence par là la propension à l'évitement. Enfin, deux composantes portées sur le schéma, qui ne concernent qu'une partie des collèges, vont constituer des facteurs aggravants. Si un processus d'embourgeoisement du quartier existe, il interviendra de deux manières: il jouera indirectement en véhiculant une certaine image négative du quartier et directement par l'évitement très fréquemment pratiqué par les "pionniers" de l'embourgeoisement ayant conservé des liens avec leur quartier d'origine. Ce processus d'embourgeoisement résidentiel entraîne un accroissement significatif de l'évitement, non seulement directement (les populations pionnières de l'embourgeoisement évitent beaucoup) mais aussi indirectement, en renforçant l'hétérogénéité sociale entre établissements car tous les quartiers populaires ne sont pas également affectés en même temps (érosion différentielle).

Fig.14 le système de l'écrémage 


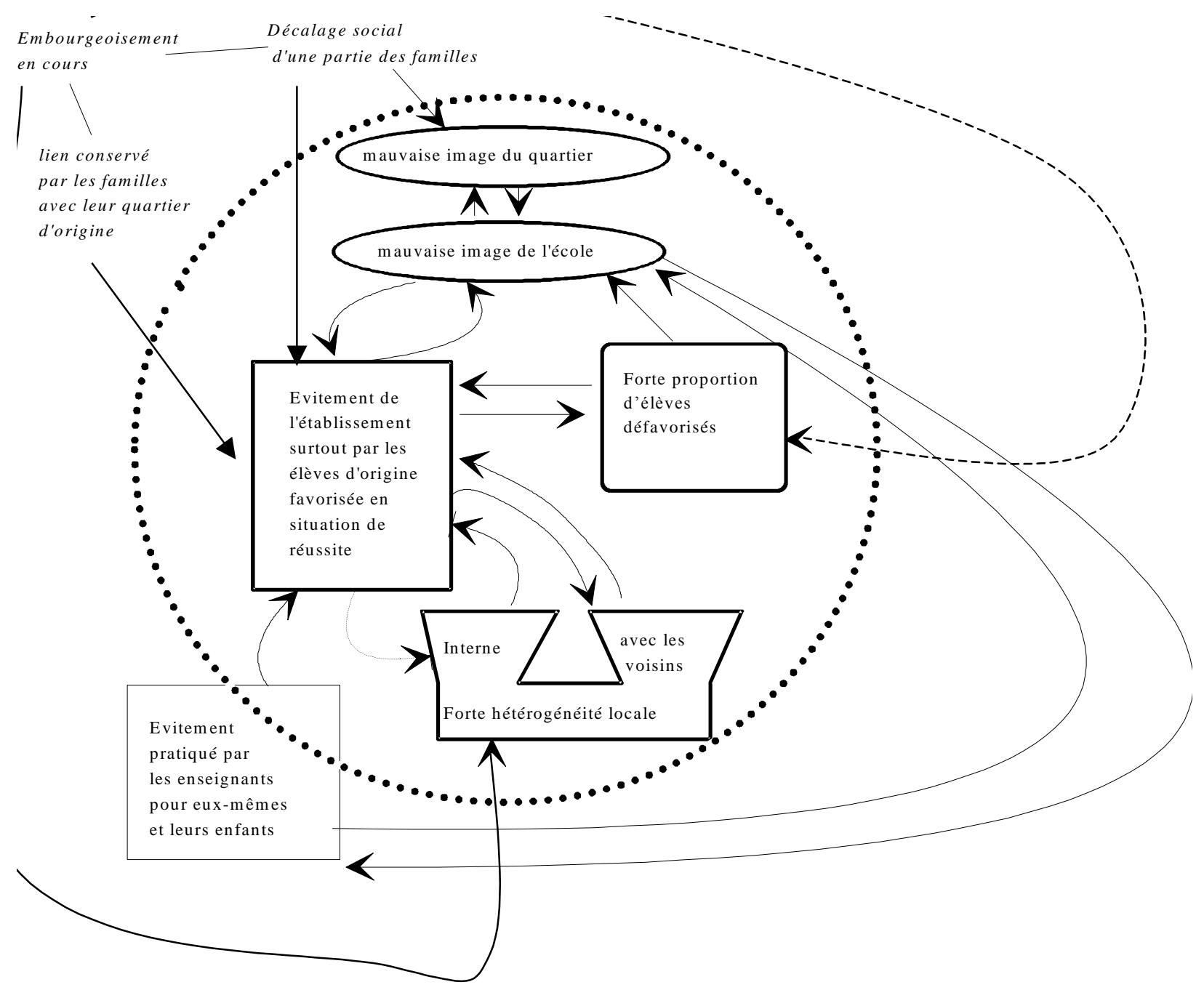

$\underline{\text { Nature de la }}$

accentue, favorise

diminue, compromet

\section{$\underline{\text { Nature des éléments }}$}

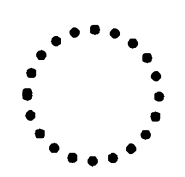

Embourgeoisement

en cours coeur du système

de l'écremage

élément exogène 


\title{
Conclusion
}

A Paris, la carte scolaire demeure, malgré l'évitement, un frein à la division sociale de l'espace. Cependant, année après année, l'efficacité de ce frein diminue à mesure que les pratiques d'évitement se diffusent tout en se diversifiant.

Si l'évitement à l'entrée en $6^{\mathrm{e}}$ est une pratique de plus en plus répandue géographiquement et socialement, il est encore pratiqué très diversement par les catégories d'élèves examinées. Ainsi, bien que les chances de réussite d'une demande de dérogation soient très peu influencées par l'origine sociale du demandeur, l'évitement a un impact mesurable sur la ségrégation sociale à l'école. Etant donnée la composition sociale réelle des secteurs de recrutement des collèges en 2001-2002, il apparait que l'évitement positif des élèves favorisés compromet davantage la mixité sociale à l'école que ne le fait l'évitement négatif vers le privé. Toutefois, en matière d'évitement négatif, le recours au privé accroît bien davantage le niveau de ségrégation que ne le fait l'évitement négatif interne au secteur public.

Ces effets sur la mixité sociale sont de peu d'importance en regard des conséquences de l'évitement sur la division sociale de l'espace scolaire, à savoir l'accroissement les contrastes, la simplification des configurations spatiales (en tendant par exemple à homogénéiser le nord parisien). Dans ce contexte, les élèves socialement favorisés évitent plus que les autres et paraissent particulièrement sensibles à la composition sociale de leur établissement de secteur. Ils sont également nettement plus mobiles, au point qu'il n'est pas possible d'établir un lien statistique entre l'environnement socio-scolaire immédiat de leur établissement de secteur et leur propension à déroger. Les élèves défavorisés, au contraire, sont rarement mobiles, et, quand ils le sont, le voisinage immédiat semble pouvoir jouer un certain rôle dans leur décision d'éviter. Les catégories moyennes, quant à elles, imitent les catégories favorisées, tout en étant bien davantage influencées par la composition sociale des établissements avoisinants (leur mobilité se fait à relativement courte portée). A l'échelon des établissements, c'est souvent le comportement de ces catégories face à l'évitement qui est déterminant. Or, plus le paysage social est simple et contrasté, plus la perception locale des différences est facile, et plus il est à craindre que l'évitement ne compromette la mixité sociale à l'école. C'est pourquoi la simplification de la géographie socio-scolaire induite par l'évitement est un signal alarmant.

\author{
REFERENCES
}

ABDALLAH-PRETCEILLE M., 1992, Quelle école pour quelle intégration? - CNDP/Hachette.

ALDEGHI, TABARD N., 1990, La ségrégation croît en Ile de France - La Lettre de l'Observatoire de la dynamique des localisations ODL-Ile de France (GIP RECLUS).

BALL S.J., BOWE R. \& GEWIRTZ S.,1995, «School choice, social class and distinction : the realization of social advantage in education », Journal of Education Policy, 11 (1), 1995, pp.89-112.

BALLION R., 1982, Les consommateurs d'école, Paris, Stock. 
BARTHELEMY M.,1995, «Des militants de l'école : les associations de parents d'élèves en France », Revue française de sociologie, XXXVI, 1995, pp. 439-472.

BARTHON C., 1996, "La scolarisation des enfants dans l'académie de Versailles : à la recherche de l'effet de contexte », Espace Population Société, n²-3 1996, p.457-466

BARTHON C., 1997, «Enfants d'immigrés au collège: intégration ou ségrégation scolaire? », In Réussite scolaire et universitaire, AUBERT F. \& Alii (eds), L'Harmatthan

BARTHON C., OBERTI M., 2000 «Ségrégation spatiale, évitement et choix des établissements », L'école : l'état des savoirs, sous la direction de A.VAN ZANTEN, La Découverte

BRIAND J.-P., CHAPOULIE J.M., 1992, Les collèges du peuple. L'enseignement primaire supérieur et de développement de la scolarisation prolongée sous la Troisième République, Paris, Editions du CNRS.

BROCCOLICHI S.,1995, "Orientations et ségrégations nouvelles dans l'enseignement secondaire », Sociétés contemporaines, $\mathrm{n}^{\circ} 21,1995$, pp.15-27.

BROCCOLICHI S., VAN ZANTEN A.,1997, « Espaces de concurrence et circuits de scolarisation. L'évitement des collèges publics dans un district de la banlieue parisienne », Annales de la recherche urbaine, n²75, 1997, pp.5-17.

BROCCOLICHI S., "Inquiétudes parentales et sens des migrations d'élèves. L'évitement croissant des collèges publics dans un district de la banlieue parisienne », Les dossiers d'éducation et formation, $\mathrm{n}^{\circ} 101$.

BRUN J., CHAUVIRE Y., 1983, La ségrégation sociale, observations critiques sur la notion et essais de mesure à partir de l'exemple de Paris (1962-1975), Géographie sociale (acte du colloque de Lyon), p.102-133

BRUN J., RHEIN C. (coord.), 1994, La ségrégation dans la ville. Concepts et mesures, Paris, L’Harmattan.

COUSIN O. 1998, L'efficacité des collèges. Sociologie de l'effet établissement, Paris, PUF.

DURU-BELlat M., MINGAT A., 1989, Carte scolaire et orientation en fin de cinquième - L'Orientation Scolaire et Professionnelle, ${ }^{\circ} 2$, p.171-184.

DURU-BELLAT M., MINGAT A., 1988, «Le déroulement de la scolarité au collège : le contexte fait des différences », Revue française de sociologie, vol.XXIX, 1988, pp.649-666.

FELOUZIS, Le collège au quotidien, Paris, PUF, 1994.

FRANÇOIS J.C., Discontinuités dans la ville: l'espace des collèges de l'agglomération parisienne 1982-1992 -

Thèse de Doctorat, Université Paris I, 1995.

FRANÇOIS J.C., "Intégration spatiale et retard scolaire dans l'espace des collèges de l'agglomération parisienne, 1982-1992 », Historiens \& Géographes, n³58, 1996.

FRANÇOIS J.C., Diffusion et dynamique des discontinuités: les élèves d'origine africaine dans l'espace des collèges de l'agglomération parisienne - Mappemonde n4/1996, pp. 31-37

FRANÇOIS J.C.,1998, «Discontinuités territoriales et mise en évidence de systèmes spatiaux dans l'espace des collèges de l'agglomération parisienne », L’Espace Géographique n¹/98, 1998, pp63-75.

FRANÇOIS J.C.,2002 ? «Les élèves d'origine étrangère dans l'espace des collèges de l'agglomération parisienne 1982-1997. Une ségrégation qui s'accrô̂t? »-L'Espace Géographique, (à paraître)

GLASMAN D., 1994, «La politique scolaire des municipalités: cohérence d'une visée d'intégration ou alibi? » - Migrants-Formation, ${ }^{\circ} 97$, p.99-109

GOUX D., MAURIN E., 1995, «Origine sociale et destinée scolaire », Revue française de sociologie, XXXVI, pp.81-121. 
GRISAY A., "Le fonctionnement des collèges et ses effets sur les élèves de $6^{\circ}$ et de $5^{\circ}$ », Education et Formation, DEP, $n^{\circ} 32$.

HENRIOT-VAN ZENTEN A., PAYET J.P., ROULLEAU-BERGER L.,1994 L'école dans la ville. Accords et désaccords autour d'un projet politique, L'Harmattan.

HERIN R., «Démocratisation de l'enseignement et diversité géographique des systèmes éducatifs », EspacePopulations-Sociétés, ${ }^{\circ} 1$, pp.11-31.

KERROUBI M., 1997, «De l'école populaire à l'école difficile : émergence du niveau '"établissement'” », in van Zanten (coord.), La scolarisation dans les milieux difficiles, Paris, INRP, 1997.

LANGOUET G., LEGER A.,1994, Ecole publique ou école privée? Trajectoires et réussites scolaires - Fabert.

LEGER A., TRIPIER M., 1986, Fuir ou construire l'école populaire ? Paris, PUF.

LEVY J. et al., «Les disparités entre collèges publics », Educations et formations, n8, pp.11-33.

PINCON M., PINCON-CHARLOT M.,1996, Les beaux quartiers, Paris, Payot.

PINCON M., PINCON-CHARLOT M.,1994, «Les enfants d'immigrés dans une école des beaux-quartiers », Migrants-Formation, ${ }^{\circ} 96,1994$, pp.73-81.

PINCON-CHARLOT M., PRETECEILLE E., RENDU P.,1986, Ségrégation urbaine, classes sociales et équipements collectifs en région parisienne, Anthropos.

POUPEAU F., 2001, Enseigner en «banlieue ». Sociologie de l'éducation et crise du système d'enseignement, thèse de doctorat, EHESS, 501p.

RHEIN C. 2001, «La notion d'intégration en sociologie», introduction au séminaire $M S^{2}$ «Intégration spatiale et intégration sociale : l'espace compte » organisé par l’UMR 8504, Paris, janvier 2001.

RHEIN C., 1997, «De l'anamorphose en géographie. Polarisation sociale et flux scolaires dans la métropole parisienne », Annales de la recherche urbaine, nº75, pp. 59-69.

RHEIN C. 1999, (avec LE PAGE A. et GROSBRAS P.-A.), Division sociale de l'espace et inégalités de scolarisation, Rapport de recherche au Plan Construction Architecture, Direction de la construction, Ministère de l'Equipement et du Logement.

TRANCART D., "L'évolution des disparités des collèges publics », Revue française de pédagogie, $\mathrm{n}^{\circ} 124$, pp.43-53.

VAN ZANTEN A.,1996, "Fabrication et effets de la ségrégation scolaire », in L'exclusion : l'état des savoirs, sous la direction de PAUGAM S., Paris, La Découverte, 1996, pp.281-291.

WOODS P. \& BAGLEY C.,1996, «Market Elements in a Public Service : an analytical model for studying educational policy », Journal of Education Policy, vol.11, n ${ }^{\circ}$, pp.641-653 